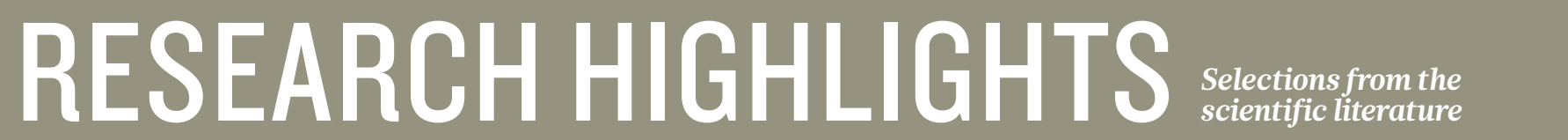

NEUROSCIENCE

\section{Transplanted brain cells calm fear}

Mice that receive neuron transplants are better at forgetting fearful memories than those without transplants.

Yong-Chun Yu at Fudan University in Shanghai, China, and his colleagues studied mice that had learned a fearful memory and were then trained to forget it. After this 'extinction' training, fear memories often come back spontaneously with time or in response to a stimulus. But the team found that this later recurrence was reduced when embryonic neurons that make a neurotransmitter called GABA were transplanted into the animals' brains two weeks before the extinction training.

The neurons were transplanted into the amygdala, a brain region associated with fear, and the findings suggest that the cells may have returned it to a more pliable, juvenile state. This could increase the effectiveness of fear-extinction training, the authors suggest. Neuron http://doi.org/bvp3 (2016)

\section{ASTROPHYSICS}

\section{Dark matter may not be so clumpy}

An analysis of almost 15 million distant galaxies reveals that dark matter may be slightly less dense and more evenly distributed throughout space than was thought.

Dark matter makes up onequarter of the Universe's mass, but is invisible and its presence can only be inferred from its gravitational effects. A team led by Hendrik Hildebrandt of the Argelander Institute for Astronomy in Bonn, Germany, and Massimo Viola of Leiden University in the Netherlands

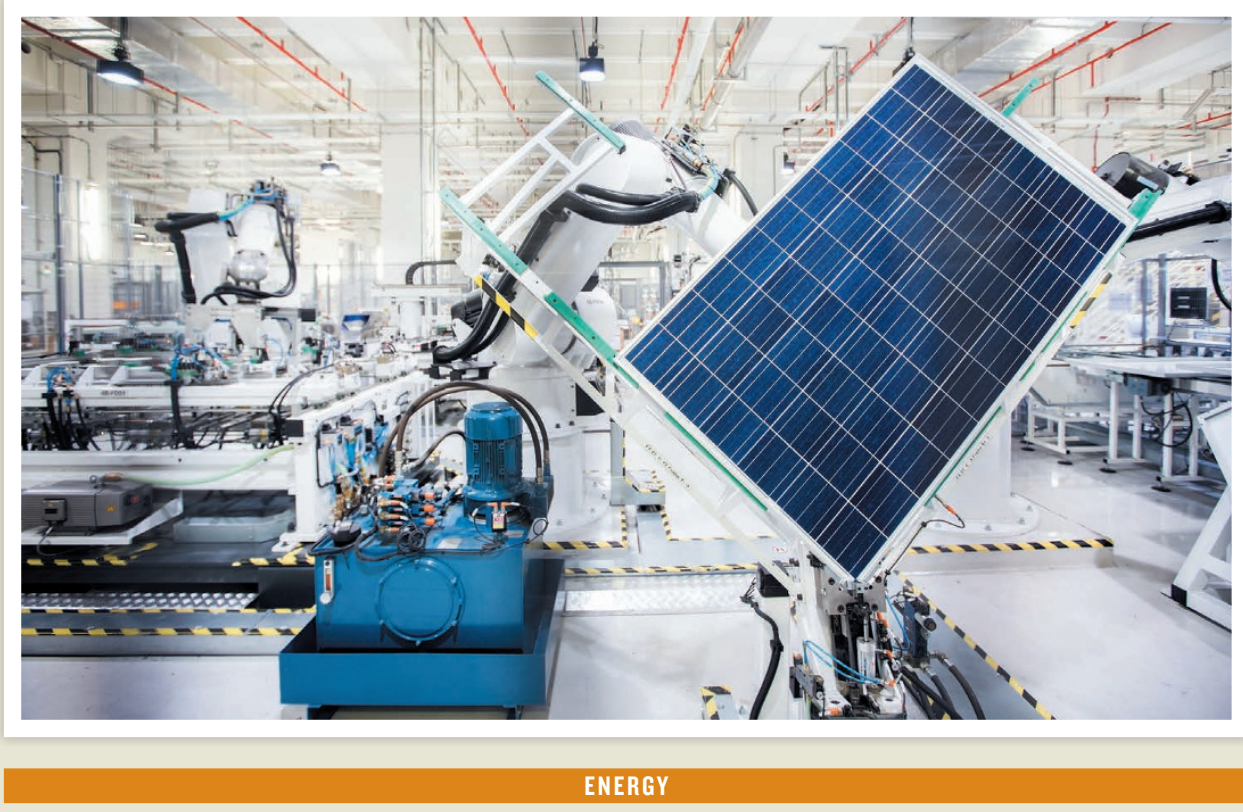

\section{Solar power pays off}

Solar-cell production generates high levels of greenhouse-gas emissions, leading some to question the environmental sustainability of the booming business (pictured) - a concern now allayed by scientists in the Netherlands.

A team led by Atse Louwen at Utrecht University studied developments in photovoltaic production around the world between 1976 and 2014. The authors found that, thanks to ongoing improvements to the technology and production methods, every doubling of global photovoltaic capacity was associated with a drop of up to $13 \%$ in the energy used during system production, and a fall of as much as $24 \%$ in greenhouse-gas emissions.

Even in the worst-case scenario, whereby solar panels perform at their lowest efficiency levels, the industry is set to break even in 2017 in terms of energy use and in 2018 for emissions. Nature Commun. 7, 13728 (2016) examined galaxy images taken by the European Southern Observatory's VLT Survey Telescope in Chile as part of the Kilo-Degree Survey. The researchers measured cosmic shear: the distortion of the shapes of background galaxies due to light that is warped by the gravitational effects of large-scale structures such as galaxy clusters. The team statistically measured how dark matter subtly distorted the galaxy images, and inferred its density from this.

If future measurements confirm this more-even distribution of dark matter, astrophysicists might need to revise their models of how the Universe evolved.

Mon. Not. R. Astron. Soc. (in the press); preprint at https://arxiv. org/abs/1606.05338 (2016)

\section{BIOMATERIALS}

\section{How additives} preserve vaccines

Scientists have found additives that, at low concentrations, extend the life of vaccines at room temperature.

High levels of sugar stabilize virus particles in vaccines, but the mechanism was unclear. Francesco Stellacci at the Swiss Federal Institute of Technology in Lausanne and his colleagues studied how sucrose and two other additive candidates affect viruses over time. They found that low concentrations of the polymer polyethylene glycol and gold nanoparticles mimicked the effects of sugar, increasing the half-life of a virus called adenovirus type 5 from 7 days to more than 30 days at room temperature.

The team concludes that high levels of sugar keep viruses structurally intact mainly by making the vaccines more viscous. For the other additives, particles act directly on the virus's protein shell to prevent it from degrading. The findings 